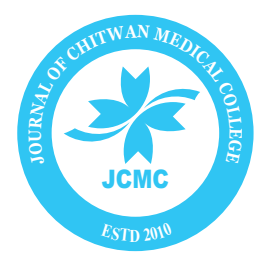

\author{
Journal of Chitwan Medical College 2019;9(30):39-42 \\ Available online at: www.jcmc.cmc.edu.np
}

\title{
EMERGENCY DEPARTMENT LENGTH OF STAY AND DISPOSAL OF PATIENTS IN CHITWAN MEDICAL COLLEGE, NEPAL
}

Daya Ram Lamsal ${ }^{1,}$, Krishna Bahadur Raut ${ }^{2}$, Parash Adhikari ${ }^{3}$

${ }^{1}$ Department of General Practice and Emergency Medicine, Chitwan Medical College, Bharatpur, Chitwan, Nepal ${ }^{2}$ Department of General Practice and Emergency Medicine, Chitwan Medical College, Bharatpur, Chitwan, Nepal ${ }^{3}$ Department of Community Medicine, Chitwan Medical College, Bharatpur, Chitwan, Nepal

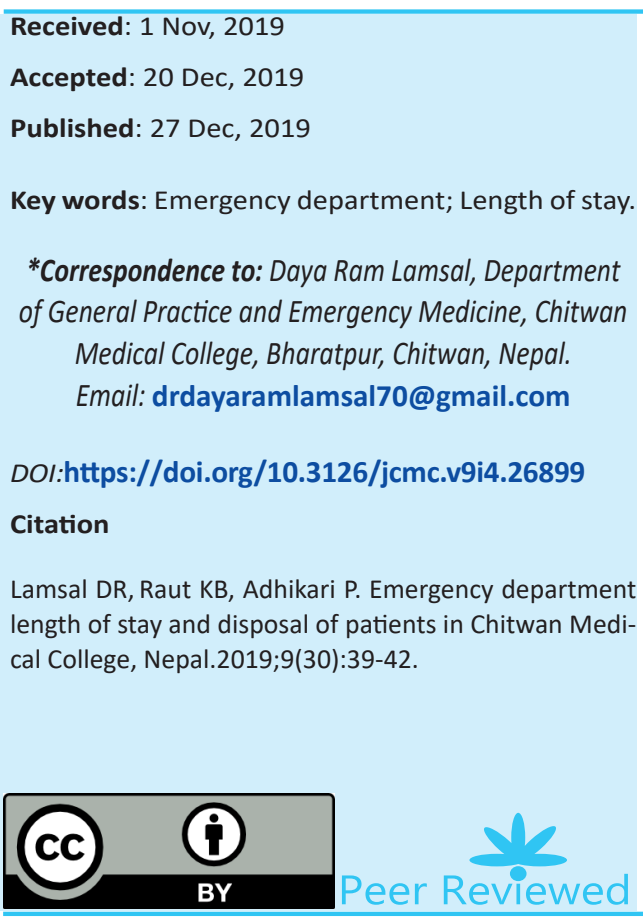

INTRODUCTION

To tackle heavy patients crowding, the United Kingdom implemented the '4-hour rule' demanding that $98 \%$ of all patients must be seen and discharged or admitted within $4 \mathrm{~h}$ of their arrival in the emergency department from the year 2004.1 Such timeframe encouraged adopting a standard waiting time policy to other countries in their National Health policy to gain improved access to emergency services and subsequent care on time. The six hours' target in New Zealand is associated with reduced mortality and greater efficiency of emergency performance.2 Strategy to enhance clearing emergency departments, National Emergency Access Target (NEST) was introduced in Australia to reduce unintended consequences.3 Studies have revealed that no single cause is associated for prolonged length of stay at emergency department.4 Debate and discussions are going on about the definitive waiting time policy as many hospitals are unable to achieve the target set by NHS trust in UK to revise the policy. 5 The organizational factors may influence the length of stay at emergency department apart from the external factors. 6 Conceptual framework on the possible delays in the ED process has been outlined by input, throughput and output where the internal and external factors has been identified to be responsible emergency stay.7 Currently time sensitive high acuity illness like myocardial infraction, stroke and sepsis protocols follow shorter management outline than four-hour protocol one of the example is one hour bundle of septic shock management in ED recommended by survival from sepsis campaign.8 Many developing countries like Nepal have no definitive waiting time national standard but trying to follow international practices feasible in the local context even though clinical and managerial protocols are still unclear in the organization itself. The study aimed to assess the Length of Stay (LOS) and disposal of patients visiting Emergency Department of Chitwan Medical College.

\section{METHODS}

This is a retrospective study carried out in the emergency department of Chitwan Medical College (CMC) from $01 / 10 / 2018$ to $30 / 09 / 2019$. All the patients reported to emergency department during this period were included in the study except the patients who arrived for delivery and the new baby admitted in hospital. Electronic medical records were obtained from medical record section. Two hundred ninety eight patient's records were not fulfilling the complete information and thus were omitted from the study. Total files 
of 29057 patients were included in the study. The length of stay was calculated from the time of arrival to disposal in the emergency department. Six-hour time frame was regarded as a standard waiting time for this study following existing operating hospital's policy. Obtained data were tabulated and analyzed. Ethical approval was obtained from the institution review committee.

Length of stay (LOS): LOS defined as the time spent in the emergency department as seen on patient's electronic case record from the time of arrival to the time of disposal.

Children age group was considered in this study from birth to 14 years (Up to the age of 14, children are treated in pediatric Department) while 60 years and above was regarded as elderly patients

\section{RESULTS}

The total patients visiting emergency department during the study period were 29057. Out of them 14867 were male, 14189 were female and 1 patient was categorized as other (third gender). Median LOS in ED was 3.41 hours with a minimum of 0.4 hours and maximum of 87.21.Among patients visited to emergency $54.57 \%$ discharged from emergency and $40.83 \%$ admitted in hospital.

Table 1: Number of Patients visited according to age group

\begin{tabular}{|l|l|l|l|}
\hline & Age group & No. of Patients & Percent \\
\hline Children & $0-14$ & 4279 & 14.7 \\
\hline Adult & $15-59$ & 18491 & 63.6 \\
\hline Elderly & 60 \& above & 6287 & 21.6 \\
\hline Total & & 29057 & 100.0 \\
\hline
\end{tabular}

Table 2: Intervals of LOS of all Patients visiting to ED

\begin{tabular}{|l|l|l|}
\hline Time interval (hours ) & No. of Patients & Percent \\
\hline$<6$ & 22387 & 77.0 \\
\hline $6-12$ & 4343 & 14.9 \\
\hline $12-18$ & 1599 & 5.5 \\
\hline $18-24$ & 542 & 1.9 \\
\hline$>=24$ & 186 & 0.6 \\
\hline Total & 29057 & 100.0 \\
\hline
\end{tabular}

Total number of patients arrived in ED $n=29057$

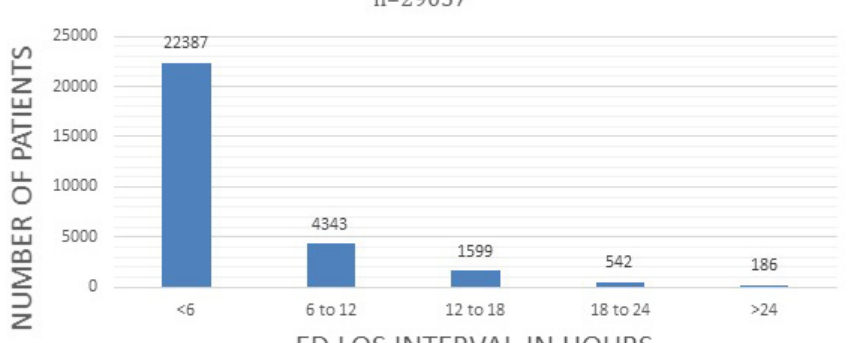

Figure 1: Diagram showing Intervals of LOS of all Patients visiting to $E D$
Table 3: Disposal of Patients from ED

\begin{tabular}{|l|l|l|}
\hline Disposal & Patients & Percentage (\%) \\
\hline Admission & 11865 & 40.83 \\
\hline Discharge Home & 15856 & 54.57 \\
\hline Refer & 327 & 1.13 \\
\hline LAMA & 660 & 2.27 \\
\hline Death & 72 & 0.25 \\
\hline Unspecified & 277 & 0.95 \\
\hline Total & 29057 & 100.00 \\
\hline
\end{tabular}

Table 4: Comparison of critical \& non-critical area of patients admitted

\begin{tabular}{|l|l|l|}
\hline $\begin{array}{l}\text { Category of } \\
\text { Admitted Area }\end{array}$ & & \\
\hline & No. of Patients & Percent \\
\hline Critical Care & 4021 & 33.89 \\
\hline General Ward & 7844 & 66.11 \\
\hline Total & 11865 & 100.0 \\
\hline
\end{tabular}

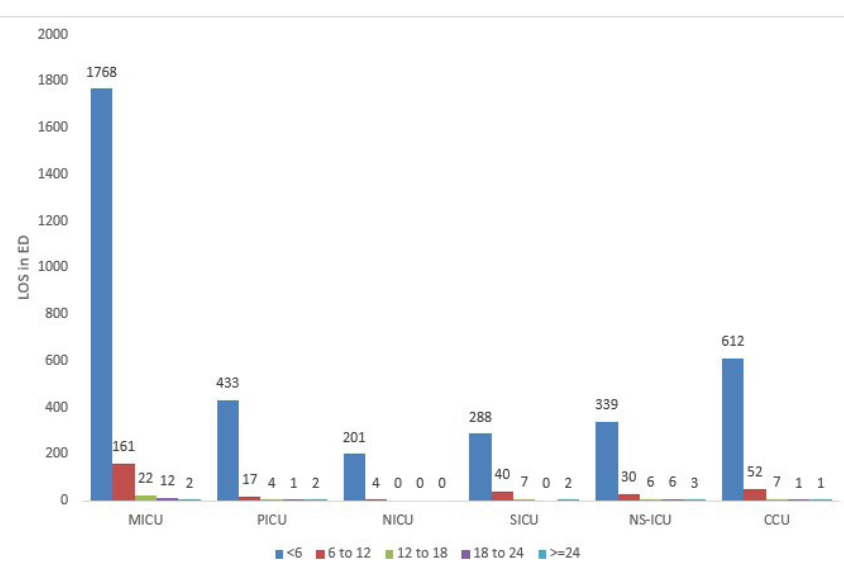

Figure 2: ED LOS in hours among patients Admitted to Critical Areas

\section{DISCUSSION}

The study shows the median length of stay (LOS) in emergency department to be 3.48 hours (with minimum of 0.4 hours and maximum of 84 ) which is quite satisfactory compared to the four policy of waiting time standard of NHS UK guideline. Such target of waiting time might lead to a positive impact on patient's outcome.9 for an instance, high performing hospitals reported a reduction in access block between $27 \%$ and $42 \%$ reduction in and improvement in the proportion of patients being seen within 4-hours between $16 \%$ and $28 \%$ in report published in New Zealand to their own performance helping to provide quality of care.10

The analysis also revealed $77 \%$ of patients leaving the ED within six hours compared to $95 \%$ observed in most of international guidelines on waiting time. 
Table 4: Comparison of critical \& non-critical area of patients admitted

\begin{tabular}{|c|c|c|c|c|c|c|c|}
\hline & & \multicolumn{5}{|c|}{ Category stay hour in emergency } & \multirow[b]{2}{*}{ Total } \\
\hline & & $<6$ & 12-Jun & 18-Dec & $18-24$ & $>=24$ & \\
\hline \multirow{3}{*}{ MICU } & Count & 1768 & 161 & 22 & 12 & 2 & 1965 \\
\hline & $\%$ within Admitted area of patients & $90.00 \%$ & $8.20 \%$ & $1.10 \%$ & $0.60 \%$ & $0.10 \%$ & $100.00 \%$ \\
\hline & $\%$ within Category stay hour in emergency & $48.60 \%$ & $53.00 \%$ & $47.80 \%$ & $60.00 \%$ & $20.00 \%$ & $48.90 \%$ \\
\hline \multirow{3}{*}{ PICU } & Count & 433 & 17 & 4 & 1 & 2 & 457 \\
\hline & $\%$ within Admitted area of patients & $94.70 \%$ & $3.70 \%$ & $0.90 \%$ & $0.20 \%$ & $0.40 \%$ & $100.00 \%$ \\
\hline & $\%$ within Category stay hour in emergency & $11.90 \%$ & $5.60 \%$ & $8.70 \%$ & $5.00 \%$ & $20.00 \%$ & $11.40 \%$ \\
\hline \multirow{3}{*}{ NICU } & Count & 201 & 4 & 0 & 0 & 0 & 205 \\
\hline & $\%$ within Admitted area of patients & $98.00 \%$ & $2.00 \%$ & $0.00 \%$ & $0.00 \%$ & $0.00 \%$ & $100.00 \%$ \\
\hline & $\%$ within Category stay hour in emergency & $5.50 \%$ & $1.30 \%$ & $0.00 \%$ & $0.00 \%$ & $0.00 \%$ & $5.10 \%$ \\
\hline \multirow{3}{*}{ SICU } & Count & 288 & 40 & 7 & 0 & 2 & 337 \\
\hline & $\%$ within Admitted area of patients & $85.50 \%$ & $11.90 \%$ & $2.10 \%$ & $0.00 \%$ & $0.60 \%$ & $100.00 \%$ \\
\hline & $\%$ within Category stay hour in emergency & $7.90 \%$ & $13.20 \%$ & $15.20 \%$ & $0.00 \%$ & $20.00 \%$ & $8.40 \%$ \\
\hline \multirow{3}{*}{ NS-ICU } & Count & 339 & 30 & 6 & 6 & 3 & 384 \\
\hline & $\%$ within Admitted area of patients & $88.30 \%$ & $7.80 \%$ & $1.60 \%$ & $1.60 \%$ & $0.80 \%$ & $100.00 \%$ \\
\hline & $\%$ within Category stay hour in emergency & $9.30 \%$ & $9.90 \%$ & $13.00 \%$ & $30.00 \%$ & $30.00 \%$ & $9.50 \%$ \\
\hline \multirow{3}{*}{$\mathrm{CCU}$} & Count & 612 & 52 & 7 & 1 & 1 & 673 \\
\hline & $\%$ within Admitted area of patients & $90.90 \%$ & $7.70 \%$ & $1.00 \%$ & $0.10 \%$ & $0.10 \%$ & $100.00 \%$ \\
\hline & \% within Category stay hour in emergency & $16.80 \%$ & $17.10 \%$ & $15.20 \%$ & $5.00 \%$ & $10.00 \%$ & $16.70 \%$ \\
\hline \multirow{3}{*}{ Total } & Count & 3641 & 304 & 46 & 20 & 10 & 4021 \\
\hline & $\%$ within Admitted area of patients & $90.50 \%$ & $7.60 \%$ & $1.10 \%$ & $0.50 \%$ & $0.20 \%$ & $100.00 \%$ \\
\hline & $\%$ within Category stay hour in emergency & $100.00 \%$ & $100.00 \%$ & $100.00 \%$ & $100.00 \%$ & $100.00 \%$ & $100.00 \%$ \\
\hline
\end{tabular}

In this study, we could not assess the delays in transfer of admitted patients from ED to inpatient department after consultation and 'boarding time' which is regarded as one of the factors associated with the prolonged length of stay and mortality.11 When the LOS was compared between the critical areas in the study population, neonatal disorder requiring admission had the shortest stay and surgical condition the longest. The reason behind it might be less time consumed in the diagnostic test for neonate and taking a longer time for preoperative diagnosis and preparation for surgical patients. Few of them staying more than six hours could be due to use of emergency observation for borderline illness or injuries.

The present study also analyzed the LOS ED to admitted patients to critical areas, most the patients were shifted to critical areas within 6 hours. An article published in North America revealed the median ED length of stay 7 (4-13) hours shorter LOS in the present review.

Medical conditions were the most common disorders requiring admission in hospital because of general teaching hospital having more resources and reputation as compared to other discipline.

Majority (54.57\%) of patients were treated and discharged from emergency department with $40.83 \%$ being admitted within the hospital. Some remaining however were referred to another hospital mostly due to unavailability of hospital beds (particularly in critical areas) or sometimes as per their wish to be shifted to another hospital due to social reasons.

Another mode of disposal revealed that $2.27 \%$ patients left emergency against doctor's advice even after counseling and written explanation; probably due to unsatisfying services. We do not have exact data as to how many patients left ED without being seen by the doctors.

\section{CONCLUSION}

The median length of stay was 3.84 hours and $54.57 \%$ of these patients were discharged after treatment with admission rate of $40.83 \%$ from Emergency department.

\section{CONFLICT OF INTEREST}

None

\section{FINANCIAL DISCLOSURE}

None 


\section{REFERENCES:}

1. Department of Health. The NHS Plan: a plan for investment, a plan for reform. Stationery Office; 2000. [Google Scholar]

2. Ardagh M. The'six hour target'in New Zealand is associated with reduced mortality and greater efficiency. The New Zealand Medical Journal (Online). 2017 May 12;130(1455):12.

3. Perera ML, Davies AW, Gnaneswaran N, Giles M, Liew D, Ritchie P, Chan ST. Clearing emergency departments and clogging wards: $N$ ational E mergency A ccess $T$ arget and the law of unintended consequences. Emergency Medicine Australasia. 2014 Dec;26(6):549-55. [DOI]

4. Campbell P, Boyle A, Higginson I. Should we scrap the target of a maximum four hour wait in emergency departments?. Bmj. 2017 Oct 25;359:j4857. [DOI]

5. Chaou CH, Chiu TF, Yen AM, Ng CJ, Chen HH. Analyzing factors affecting emergency department length of stay-using a competing risk-accelerated failure time model. Medicine. 2016 Apr;95(14). [DOI]

6. Brouns SH, Stassen PM, Lambooij SL, Dieleman J, Vanderfeesten IT, Haak HR. Organisational factors induce prolonged emergency department length of stay in elderly patients-a retrospective cohort study. PloS one.

\section{Aug 12;10(8):e0135066. [DOI]}

7. Milano PK, Desai SA, Eiting EA, Hofmann EF, Lam CN, Menchine M. Sepsis Bundle Adherence Is Associated with Improved Survival in Severe Sepsis or Septic Shock. Western Journal of Emergency Medicine. 2018 Sep;19(5):774. [DOI]

8. Surviving from sepsis Campaign :one hour bundle [LINK]

9. Salehi L, Phalpher P, Valani R, Meaney C, Amin Q, Ferrari K, Mercuri M Emergency department boarding: a descriptive analysis and measurement of impact on outcomes. Canadian Journal of Emergency Medicine. 2018 Nov;20(6):929-37. [DOI]

10. Jones P, Wells L, Harper A, Le Fevre J, Stewart J, Curtis E, Reid MJ, Ameratunga S. Impact of a national time target for ED length of stay on patient outcomes.The New Zealand Medical Journal. 2017 May; 130(1455):15-34 [PMID]

11. Shen $Y$, Lee LH. Improving the wait time to consultation at the emergency department. BMJ open quality. 2018 Jan 1;7(1):e000131. [DOI]

12. Rose L, Scales DC, Atzema C, Burns KE, Gray S, Doing C, Kiss A, Rubenfeld $G$, Lee JS. Emergency department length of stay for critical care admissions. A population-based study. Annals of the American Thoracic Society. 2016 Aug;13(8):1324-32. [DOI] 